\title{
Economical and compact briefcase spectral-domain optical coherence tomography system for primary care and point-of-care applications
}

Roshan Dsouza

Jungeun Won

Guillermo L. Monroy

Darold R. Spillman, Jr.

Stephen A. Boppart 


\title{
Economical and compact briefcase spectral-domain optical coherence tomography system for primary care and point-of-care applications
}

\author{
Roshan Dsouza, ${ }^{a}$ Jungeun Won, ${ }^{a, b}$ Guillermo L. Monroy, ${ }^{a, b}$ Darold R. Spillman Jr., ${ }^{a}$ and \\ Stephen A. Boppart ${ }^{a, b, c, d, *}$ \\ aUniversity of Illinois at Urbana-Champaign, Beckman Institute for Advanced Science and Technology, Urbana, Illinois, United States \\ bUniversity of Illinois at Urbana-Champaign, Department of Bioengineering, Urbana, Illinois, United States \\ 'University of Illinois at Urbana-Champaign, Department of Electrical and Computer Engineering, Urbana, Illinois, United States \\ dUniversity of Illinois at Urbana-Champaign, Carle-Illinois College of Medicine, Urbana, Illinois, United States
}

\begin{abstract}
Development of low-cost and portable optical coherence tomography (OCT) systems is of global interest in the OCT research community. Such systems enable utility broadly throughout a clinical facility, or in remote areas that often lack clinical infrastructure. We report the development and validation of a low-cost, portable briefcase spectral-domain optical coherence tomography (SD-OCT) system for point-of-care diagnostics in primary care centers and/or in remote settings. The self-contained briefcase OCT contains all associated optical hardware, including light source, spectrometer, hand-held probe, and a laptop. Additionally, this system utilizes unique real-time mosaicking of surface video images that are synchronized with rapid A-scan acquisition to eliminate the need for lateral scanning hardware, and enable the construction of cross-sectional B-mode images over extended lateral distances. The entire briefcase system weighs $9 \mathrm{~kg}$ and costs $\sim$ USD8000 using off-the-shelf components. System performance was validated by acquiring images of in vivo human skin on the fingertip, palm, and nail fold. The efficiency, portability, and low-cost enable accessibility and utility in primary care centers and low-resource settings. @ 2018 Society of Photo-Optical Instrumentation Engineers (SPIE) [DOI: 10.1117/1 .JBO.23.9.096003]
\end{abstract}

Keywords: optical coherence tomography; handheld; diagnostics; point-of-care; system development; spectrometers.

Paper 180158RR received Mar. 14, 2018; accepted for publication Aug. 29, 2018; published online Sep. 24, 2018.

\section{Introduction}

Optical coherence tomography (OCT) is a cross-sectional imaging modality that collects backscattered near-infrared light from the specimen. ${ }^{1}$ OCT has been explored for its potential capability of being used in clinics for noninvasive and high-resolution real-time imaging. The two main variants of OCT systems are time-domain (TD) OCT and Fourier-domain (FD) OCT. TDOCT systems operate by scanning the reference mirror at a constant velocity but have limited scan rates and sensitivity. ${ }^{1}$ This limitation was overcome through the development of FD-OCT systems to acquire depth-resolved information without moving the reference mirror. Furthermore, FD-OCT systems are further classified as spectral-domain (SD) OCT, where depth-resolved information is obtained by a spectrometer, ${ }^{2}$ and swept-source (SS) OCT that uses a tunable light source and detector to measure interference signal as a function of time. ${ }^{3}$ Significant developments in improving OCT over the past two decades have revolutionized clinical diagnostic applications. ${ }^{4}$ To date, OCT is one of the standard diagnostic procedures used for ophthalmic examinations..$^{5}$ OCT has also had broad impact in cardiology, gastroenterology, dermatology, and otolaryngology, to name only a few specialties.

Advances in an imaging system design, along with ergonomic and aesthetic delivery instrumentation such as handheld OCT probes, has led to the use of OCT in intraoperative and primary care settings. ${ }^{6,7}$ The shift of OCT from an optical

*Address all correspondence to: Stephen A. Boppart, E-mail: boppart@illinois .edu bench to a portable cart-based system was a significant endeavor and took multiple generations of system designs to improve upon portability and performance. ${ }^{8}$ Many portable OCT systems were developed using FD technologies (SD-OCT and SS-OCT), which inherently are known to have higher system performance over TD-OCT. These portable OCT systems have been used in intraoperative applications to differentiate tumor margins, ${ }^{9-11}$ in ophthalmology for imaging parafoveal photoreceptor structure in infants and children, ${ }^{12}$ and in the primary care setting to diagnose and characterize otitis media $(\mathrm{OM})$, a common ear infection in children. ${ }^{13-19}$ Despite the clinical significance of these studies, these systems are often too expensive, and their use in primary care centers and low-resource settings necessitates cost effective, compact, and portable OCT systems. Development of such systems can thus provide more widespread access to technology that has the potential to improve healthcare.

Globally, researchers are developing cost-effective and mobile OCT systems based on both TD-OCT and FD-OCT platforms with unique detection/illumination schemes. Previous reports demonstrated the development of a compact platform within a handheld low-coherence interferometry (LCI) system, ${ }^{20}$ and a multiple reference (MR) OCT system. ${ }^{21-23}$ The handheld LCI system used for in vivo ear imaging was based on a linear OCT approach, ${ }^{20}$ and the overall cost to develop this system was significantly lower than most other FD-OCT systems. MR-OCT, another low-cost OCT approach, was developed based on a partial mirror and actuator configuration, generating

$1083-3668 / 2018 / \$ 25.00$ @) 2018 SPIE 
a recirculating optical delay line. The cost of the MR-OCT engine is anticipated to be similar to that of a CD/DVD unit (USD10). ${ }^{22}$ Despite the low-cost and portability of these units, they represent only one part of a complete OCT system, which also includes other optical hardware, a processing computer and software, and a beam delivery system such as a handheld probe.

It is even envisioned that in the future, a miniaturized OCT system would be marketed as a self-diagnostic kit for consumers and would be available at local pharmacies where other home diagnostic kits are readily available. ${ }^{24}$ Several published works have shown the development of FD-OCT components on a silicon photonics platform, which might enable significant mass production in the future, resulting in a cost-effective, miniature OCT system. ${ }^{25-27}$ Several research groups have developed on-chip interferometer platforms, grating couplers, a spectrometer without a line-scan camera, a silicon photonics-integrated receiver, and a single platform interferometer with receiver. ${ }^{25-30}$ Although none of the reported studies have included all these components in one platform to date, when fully integrated, there is a great potential to significantly reduce the unit cost. A recent publication on a portable low-cost OCT system showed a fivefold cost reduction in comparison with a high-end OCT system. ${ }^{31}$ This system had a line-rate of $8.8 \mathrm{kHz}$ and the OCT images presented were comparable with a standard OCT system. The cross-sectional images were obtained using a microelectro-mechanical systems (MEMS) scanner and a liquid lens in the sample arm path to adjust the depth of focus. However, we note that this system does not include a display unit (monitor), which will add to the overall form factor, and the MEMS scanner restricts lateral scan ranges and field of view (FOV).

Here, we report a compact, portable, briefcase SD-OCT system with a handheld probe for ready use in primary care and/or point-of-care settings. The fully self-contained OCT system includes all optical hardware, handheld probe, and a laptop in a compact briefcase. This design incorporates manual lateral scanning in real time to reveal cross-sectional structural information, coupled with and synchronized to the visualization of tissue surfaces by mosaicking images. The manual lateral scanning approach not only reduces system cost and weight but also facilitates cross-sectional imaging over larger areas of tissue, and subsequently larger FOV, which otherwise would be restricted with mechanical scanning methods. We tested the performance of our system by imaging in vivo human skin and the mosaicked surface features of a dollar bill.

\section{Methods}

\subsection{Compact Optical Coherence Tomography Spectrometer}

The spectrometer in a conventional SD-OCT system is one of the most critical components, where light is dispersed onto a line scan camera to spectrally separate and measure the interferometric signal. Commercially available spectrometers demonstrate excellent performance; however, such spectrometers are often bulky and expensive, limiting their use in low-cost applications. With this regard, we developed an economical and compact spectrometer solution for SD-OCT by modifying a commercially available device (I-MON 835 OEM, Ibsen Photonics, Denmark). This spectrometer had a spectral bandwidth of $50 \mathrm{~nm}$ $(810-860 \mathrm{~nm})$ with high spectral resolution $(0.08 \mathrm{~nm})$. The OEM unit had dimensions of 23 -mm height $\times 58$-mm width $\times$ 76-mm depth. However, the included detector (ILX511B,
2048 pixels, Sony) had a low line-rate of only $100 \mathrm{~Hz}$, hindering its application in SD-OCT as the low acquisition rate would result in motion artifacts and fringe washout during in vivo imaging. To address this, the detector was replaced with a more suitable USB 2.0 charge-coupled device (CCD) line-scan camera (TCE-1024-U, 14- $\mu$ m pixel size, 1024 pixels, Mightex). This camera can operate in either an 8- or 12-bit mode and provides a line rate of either $25 \mathrm{kHz}$ or $10 \mathrm{kHz}$. For this study, this camera was operated at a $10-\mathrm{kHz}$ acquisition rate and in 12-bit mode.

A schematic of the modified spectrometer is shown in Fig. 1(a). The light from the grating had a beam spread of $12 \mathrm{~mm}$ and was matched closely to the detector array size $(14.3 \mathrm{~mm})$. Figure 1(b) shows the measured source spectrum versus the spectrometer output, confirming that the entire source spectrum was detected by the spectrometer sensor. The final dimensions of the modified spectrometer were approximately 90-mm height $\times 90$-mm width $\times 70$-mm depth.

\subsection{Briefcase Optical Coherence Tomography System}

We developed and designed this OCT system to fit into a standard briefcase (34-cm height $\times 46-\mathrm{cm}$ width $\times 13$-cm depth) to be compact, easily portable, and able to be used for point-ofcare applications (Fig. 2). Additionally, this briefcase enclosure also protects all components during transportation. The SDOCT system was based on a fiber optic Michelson interferometer configuration. Light from an uncooled superluminescent diode [Exalos, EBD270005-03, $\lambda_{c}=840 \mathrm{~nm}, \Delta \lambda=50-\mathrm{nm}$ full-width-half-maximum (FWHM), and 3.4-mW output power] was guided into one port of a $2 \times 2$ fiber coupler and split into the reference and sample arms.

In the sample arm, a handheld probe was connected via a 2-m-long fiber optic cable. The handheld probe utilized a mirror and a focusing lens $(f=60 \mathrm{~mm})$ to focus the beam on a sample. A compact high-speed USB 3.0 CCD camera (XIMEA MQ013CG-ON, 1.3 MP, 500 FPS) was also integrated into the handheld probe for concurrent acquisition of surface video images and mosaicking in real time. Light for surface illumination was provided by a high-power light-emitting diode that was coupled to an integrated fiber bundle. Light exited the distal fiber ends that were located in the nose cone of the probe. For acquisition of cross-sectional images, samples were manually scanned using the probe, and images were constructed based on the decorrelation of sequentially acquired A-scans. ${ }^{32}$ The incident optical power was $\sim 1.3 \mathrm{~mW}$, which is well below the ANSI maximum permissible exposure (MPE) limit for skin. ${ }^{33}$

In the reference arm, a variable neutral density filter was placed before the lens $(f=60 \mathrm{~mm})$ to optimize system sensitivity. The light reflected from both arms was recombined in the $2 \times 2$ fiber coupler, and the resulting spectral interference signal was captured by the modified compact OCT spectrometer. Spectral data from the line scan CCD camera of the spectrometer were acquired by a laptop (HP OMEN, CE019DX, i7-7700HQ, 8 GB RAM) via a USB 2.0 connection, sampled, and rescaled as a function of the wavenumber to display A-scans in real time. During image acquisition, the line rate of the CCD camera was set to $10 \mathrm{kHz}$ and the camera exposure time was set to $100 \mu \mathrm{s}$. The complete cost of this briefcase OCT system, including the surface imaging module, was USD8000 (Table 1), and the entire system weighed around $9 \mathrm{~kg}(20 \mathrm{lbs}$.). The cost of parts for the system was based on off-the-shelf prices for 
(a)

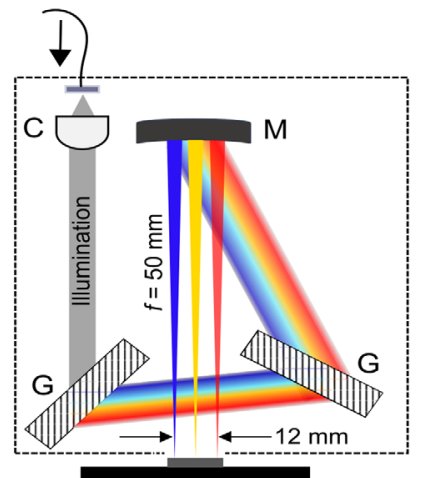

Mightex CCD camera 1024 pixels, $14 \mu \mathrm{m}$ pitch $25 \mathrm{kHz} / 10 \mathrm{kHz}$ (8/12-bit mode)

(b)

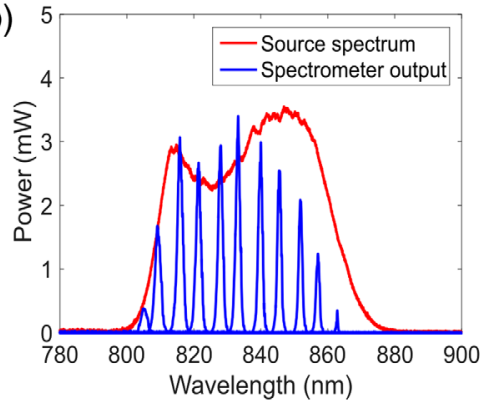

(c)

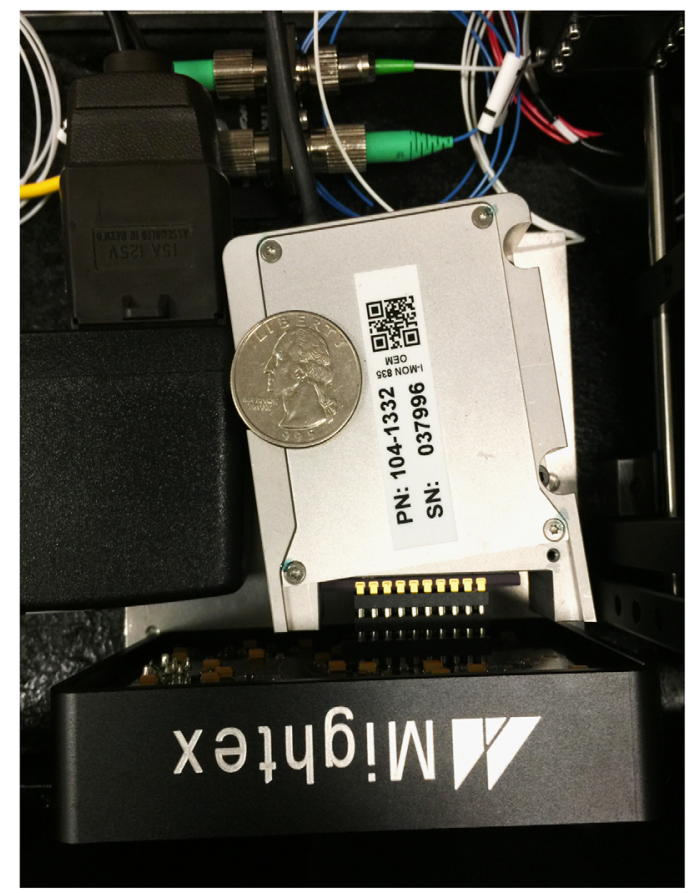

Fig. 1 Compact, high-resolution spectrometer for OCT applications. (a) Modified compact spectrometer with OEM unit (Ibsen Photonics) and CCD camera (Mightex), (b) measured spectrometer output versus source spectrum, and (c) photograph of modified spectrometer. A US quarter is shown for scale. G, grating; C, collimator; and M, concave mirror.
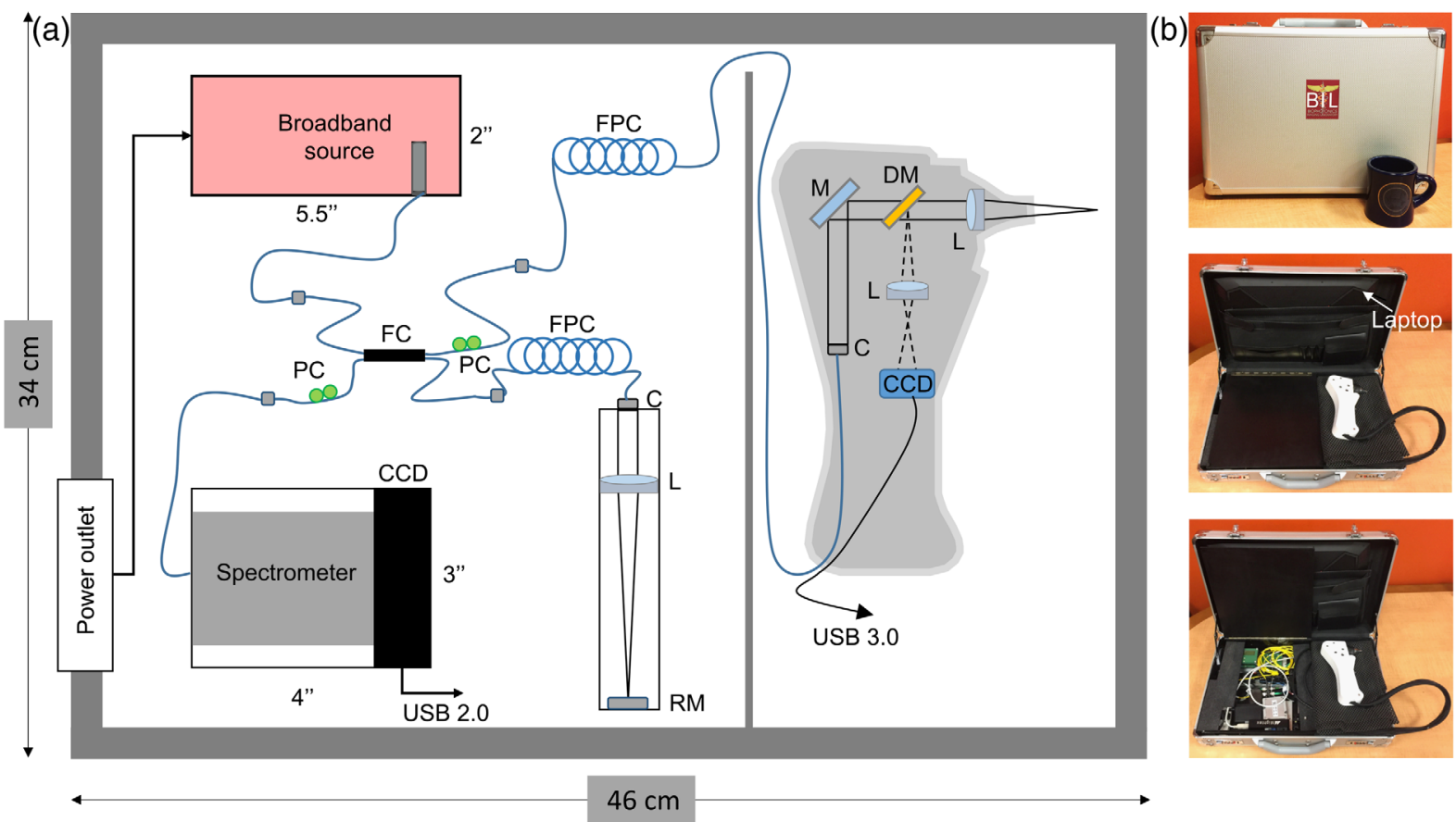

Fig. 2 Compact briefcase OCT system. (a) Schematic of the system and (b) photographs depicting the exterior and interior views of the briefcase. The top panel shows the fully enclosed briefcase OCT system. A coffee mug is shown for visual representation of the briefcase dimensions. SLD, superluminescent diode; PC, polarization controller; C, collimator; FC, fiber coupler; FPC, fiber patch cable; DM, dichroic mirror; L, lens; RM, reference mirror; and CCD, charge-coupled device camera. 
Table 1 Cost of briefcase OCT system.

\begin{tabular}{lcc} 
Component & Vendor & Cost (USD) \\
\hline OCT system & & \\
SLD + driver (1) & Exalos & $\$ 1100$ \\
$\begin{array}{l}\text { OEM interrogation } \\
\text { monitor (1) }\end{array}$ & $\begin{array}{c}\text { lbsen Photonics } \\
\text { (without detector) }\end{array}$ & $\$ 2600$ \\
Line-scan CCD camera (1) & Mightex & $\$ 1400$ \\
Fiber collimator (2) & Thorlabs & $\$ 300$ \\
Adapter for collimator (2) & Thorlabs & $\$ 59.10$ \\
Fiber coupler (1) & Thorlabs & $\$ 276$ \\
Polarization paddle (2) & Thorlabs & $\$ 394$ \\
Achromatic doublet (1) & Thorlabs & $\$ 87.25$ \\
Achromatic doublet (1) & Edmund Optics & $\$ 70$ \\
Lens mount (12) & Thorlabs & $\$ 52.50$ \\
Mirror (1) & Thorlabs & $\$ 32.60$ \\
Precision kinematic \\
mirror mount (1)
\end{tabular}

a consumer, and even lower costs are possible by further integrating components and purchasing components in volume.

Figure 3 shows photographs of the portable briefcase OCT system along with a high-end, cart-based low-coherence interferometry (LCI) system [Fig. 3(a)] previously developed by our group and used in several clinical studies. ${ }^{17,18}$ For comparison, the dimensions of the cart-based LCI system were $107-\mathrm{cm}$ height $\times 46-\mathrm{cm}$ width $\times 66-\mathrm{cm}$ depth and cost $\sim$ USD50,000. Figures 3(b) and 3(c) show the portability of the briefcase OCT system where a user/physician can easily carry the system to the primary care and/or remote setting. Photographs of human subjects and operators were obtained and used with their consent.

\subsection{Real-Time Mosaicking and B-scan Formation}

The algorithm used for OCT acquisition, image assembly, and surface image mosaicking is shown in the flowchart in Fig. 4. The processing was performed using a custom-designed graphical user interface (LabVIEW 2015) on a laptop. Initially, background spectra and resampling indices for linearization in the wave number were precalculated in the initialization step. Standard OCT processing was then performed by background subtraction, cubic B-spline interpolation for linearization, and dispersion compensation. This was followed by A-scan reconstruction by taking the Fourier transform of the recorded spectral signal. Reconstruction of the cross-sectional images was implemented with a manual scanning approach previously reported by our group. ${ }^{32}$ The use of this manual scanning method to generate cross-sectional images was implemented to minimize cost, to eliminate a lateral scanning mechanism, and to enable imaging over larger areas of tissues.

A-scan assembly was based on a defined cross-correlation threshold value set by the user, where the cross-correlation of two sequential A-scans ( $i$ and $i+1$, where $i$ corresponds to the first A-scan) is computed. The A-scans are appended to the assembled images when the correlation coefficient falls below the threshold (implying that the new A-scan is from a new lateral location), and excluded from the assembled images when the correlation coefficient is greater than the threshold (implying that the new A-scan is from the same lateral location). The decorrelation of the A-scans effectively confirms displacement of the probe beam or a change in the sample structure, which otherwise would have a higher correlation coefficient. A threshold value for the correlation coefficient in this study was empirically set to 0.8 for image assembly.

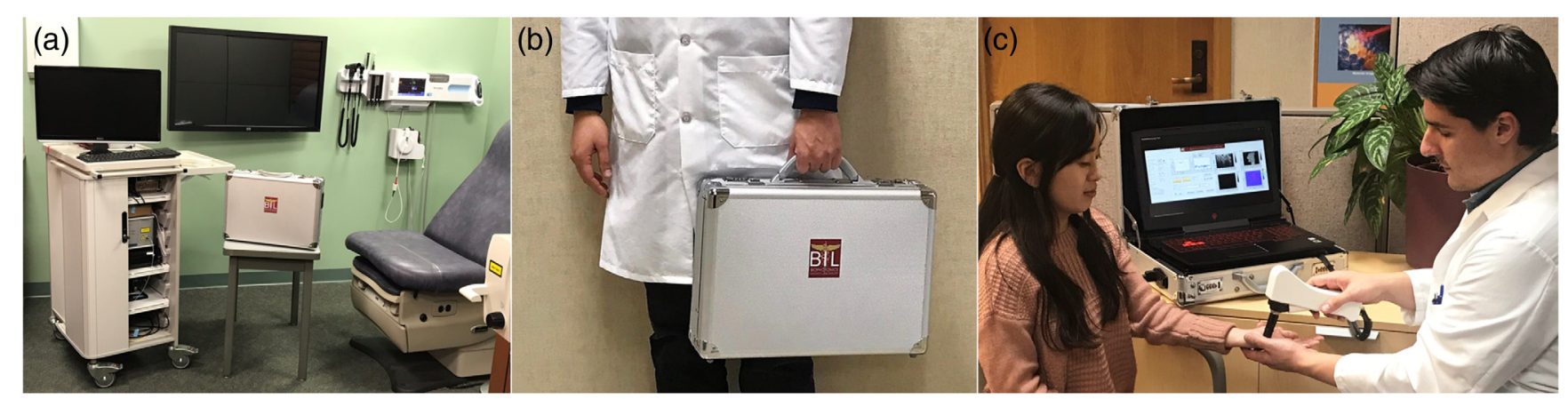

Fig. 3 Portability of the briefcase OCT system. (a) Comparison of the briefcase OCT system with a highend, cart-based LCI system. (b-c) Photographs illustrating the portability of briefcase OCT where a physician can easily carry such system for examination in primary care and point-of-care settings. 


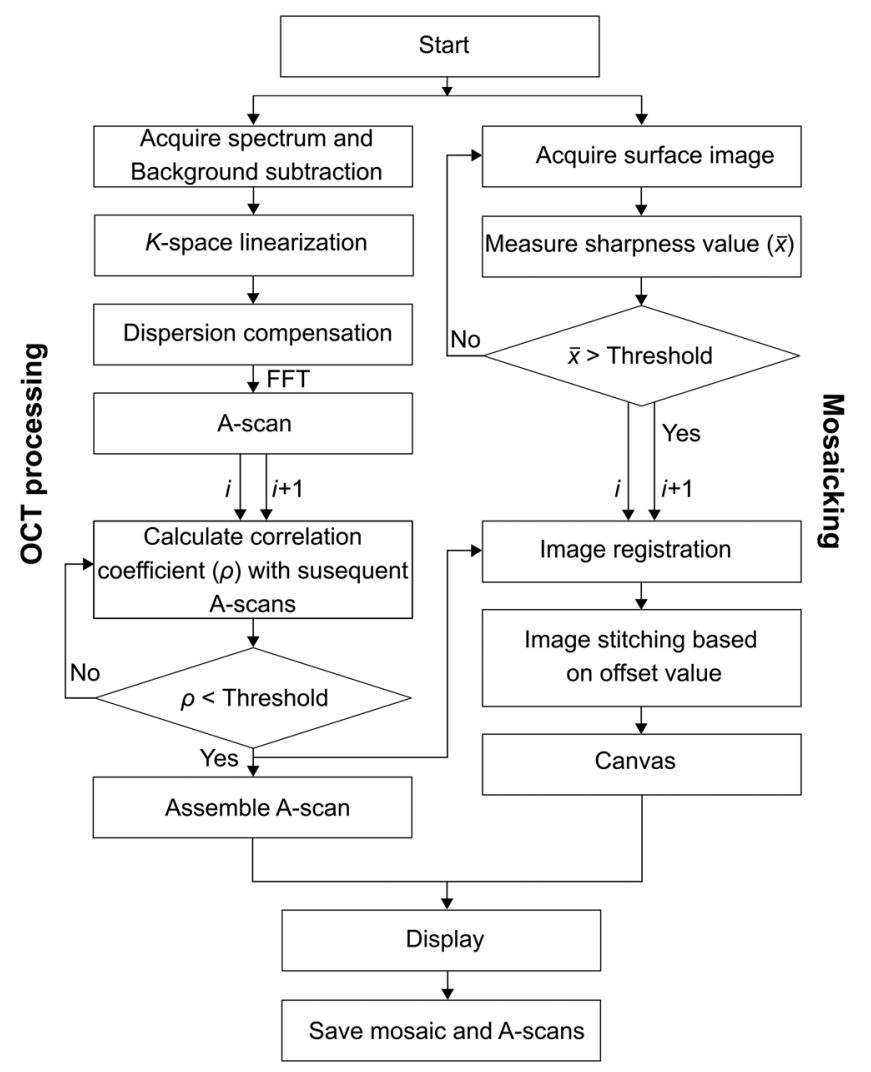

Fig. 4 Flowchart representation of the real-time OCT acquisition and surface image mosaicking. The algorithm performs OCT acquisition, B-scan formation based on the correlation coefficients, and mosaicking of surface images all in real time.
The developed algorithm also simultaneously acquires surface images of the sample and performs mosaicking in real time. For efficient data flow, a producer/consumer architecture in LabVIEW was implemented where the producer loop acquires surface images and the consumer loop does the mosaicking of acquired images in real time. The consumer loop starts only when the correlation coefficient is below the threshold level and is notified using a notifier (Fig. 4). The notifier also synchronizes the acquired A-scans for each surface image in the mosaic. This allows for the determination of the lateral two-dimensional (2-D) position of the OCT beam, and subsequently for more standard B-scan images to be extracted from regions of straight paths identified from within the total path of the beam.

For real-time mosaicking, out-of-focus or blurred images were eliminated to form a high-quality mosaic. Blurry images could also result in an error in the registration algorithm. Our algorithm incorporated a command to eliminate out-of-focus images in real time by measuring the sharpness of the image. The sharpness value (or mean value, $\bar{x}$ ) of the image was measured by taking the mean of the convoluted image using a linear filter, which effectively highlighted the features of the image. For real-time processing, the threshold value was set to correspond to the sharpness value at a depth of 0 and $1.5 \mathrm{~mm}$ [Fig. 5(d)], and any images acquired outside of these depths were excluded.

The surface image registration algorithm determines the translational offset between two consecutively acquired frames. A fast registration algorithm was implemented as this approach requires a small fraction of computational time and memory requirement. ${ }^{34,35}$ The fast registration algorithm performed a cross-correlation using the discrete Fourier transform between
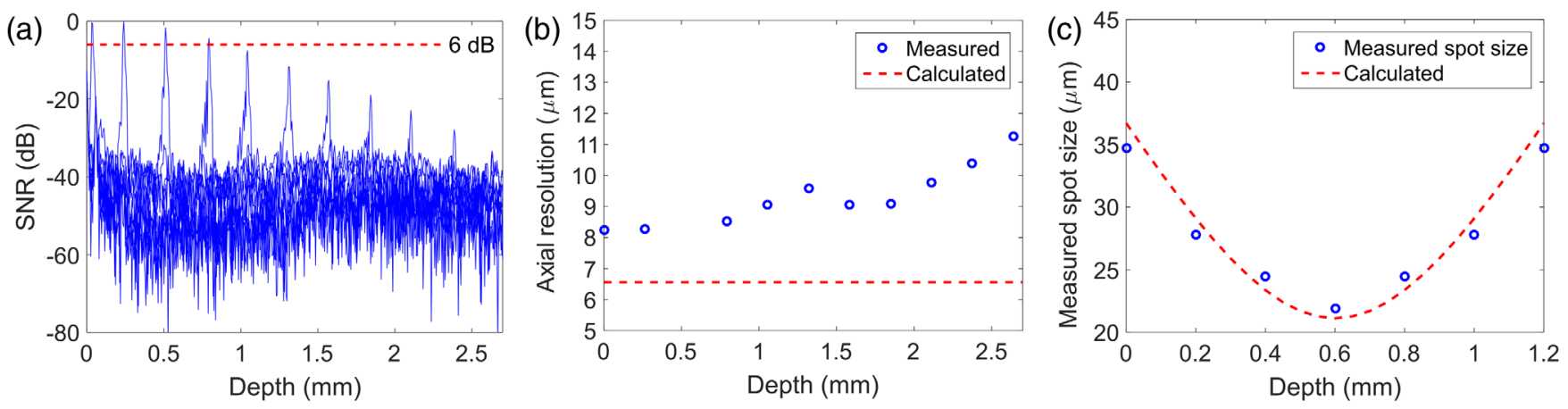

Sharpness value
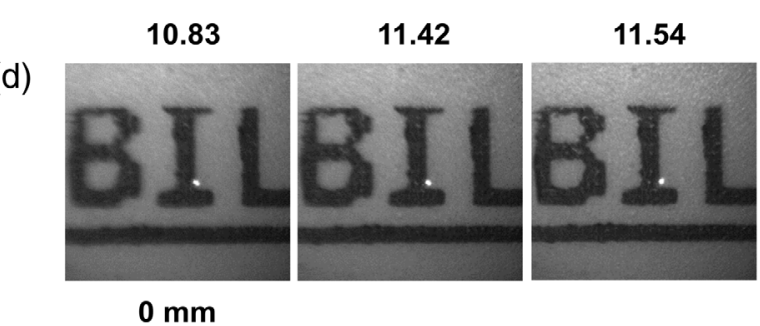

12.31

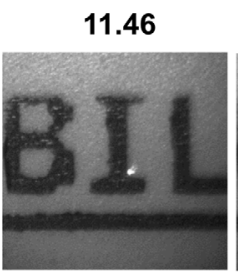

10.06

9.82
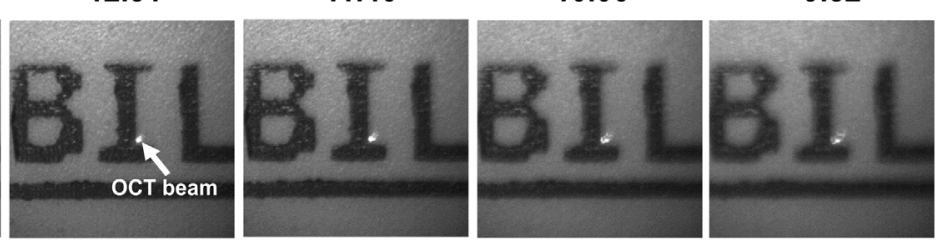

$600 \mu \mathrm{m}$

$1.2 \mathrm{~mm}$

Depth (mm)

Fig. 5 Characterization of the briefcase OCT system. (a) Measured system sensitivity roll-off using a fixed optical density filter in the sample arm. (b) Measured axial resolution at FWHM of the point spread function. (c) Lateral resolution over various depths measured with a beam profiler and compared with calculated values. (d) Representative surface images and the corresponding sharpness value used for discarding out-of-focus images in real-time. 
consecutive images. The registered images were subsequently inserted into a predefined zero matrix (also called a canvas), where the first image is copied to the center of the canvas and subsequent frames are copied with an offset value. The stitching of images was based on the dead-leaf approach, ${ }^{36}$ where a new frame overwrites an existing frame. The canvas size was approximately five times larger (FOV: $15 \mathrm{~mm} \times 15 \mathrm{~mm}$ ) than a singly acquired frame, and the time required for image registration was $\sim 10 \mathrm{~ms}$. The program saved the surface image mosaics as 12-bit PNG images, and the assembled A-scans both as raw data and as a 12-bit PNG image. The real-time data procssing time of the consumer loop was $\sim 15 \mathrm{~ms}$. The measured loop time is system (laptop) dependent and mainly relies on the usage of the CPU during data acquisition.

\section{Results}

\subsection{System Characterization}

The sensitivity of the system was measured by placing a mirror and an optical density filter $(\mathrm{OD}=2.1$, round trip loss $42 \mathrm{~dB}$ ) in the sample arm beam. Figure 5(a) shows the measured sensitivity of the system to a depth of $2.7 \mathrm{~mm}$ in air. The measured sensitivity at zero optical path difference (OPD) was $\sim 98.89 \mathrm{~dB}$, and dropped by $6 \mathrm{~dB}$ at a depth of $1 \mathrm{~mm}$ in air. For in vivo imaging (shown later), a depth range of $1.5 \mathrm{~mm}(85 \mathrm{~dB})$ was used as the sensitivity was degraded over larger depths and was closer to the noise floor. Furthermore, the low optical power from the uncooled SLD that is incident on scattering samples will limit the sensitivity of the system, which will degrade with increased A-scan rate. The OCT images were acquired at a $10 \mathrm{kHz} \mathrm{A-scan}$ rate and the image quality was sufficient to differentiate the structural features. We measured the output power over several hours (at different intervals) and found minimal fluctuations (microwatts) in output power. Future studies will investigate the dependence of output power on ambient temperature fluctuations.

Figure 5(b) shows the measured and calculated axial resolutions of the system. The calculated axial resolution based on the SLD specifications from the vendor was around $6.2 \mu \mathrm{m}$. The axial resolution was measured at the FWHM of the point spread function and was around $8.1 \mu \mathrm{m}$ at zero OPD. The $2-\mu \mathrm{m}$ discrepancy in the axial resolution may be due to slight uncertainty in dispersion correction. The measured axial resolution deteriorates with larger imaging depths (over $2 \mathrm{~mm}$ ). Nonetheless, for an imaging range up to $1.5 \mathrm{~mm}$, the system had minimal degradation compared with the zero OPD. Figure 5(c) shows the measured and calculated lateral resolutions of the system. The calculated lateral resolution was based on the focal length of the objective $(f=60 \mathrm{~mm})$ and the measured beam diameter before the objective $(3 \mathrm{~mm})$ and was around $21.4 \mu \mathrm{m}$. For imaging, we placed the focus roughly at $600-\mu \mathrm{m}$ depth. The Gaussian beam spot size (lateral resolution) was measured using a beam profiler (Thorlabs, BP209-VIS) as depicted.

The developed system will be used for imaging various tissue sites that are commonly examined in primary care clinical settings, such as the tympanic membrane of the ear. Considering this application, a longer working distance would be required, and the subsequently lower (poorer) lateral resolution would be an inherent trade-off. Further, in the designed handheld probe, the lens was fixed inside the 3-D printed case, which along with a speculum tip, comprised a total length of $\sim 55 \mathrm{~mm}$. Hence, a lens with a $60-\mathrm{mm}$ focal length was used. The reported system is not completely optimized for tympanic membrane (TM) imaging; however, we are working to optimize this system, along with improving the lateral resolution of the probe. Figure 5(d) shows the representative surface images and sharpness values for the corresponding depths, and lateral resolutions are shown in Fig. 5(c). At focus, the image demonstrated a maximum sharpness value $(\bar{x})$ that decreases away from focus.

\subsection{Cross-Sectional Imaging Using a Motorized Translation Stage}

To first test the feasibility of cross-sectional imaging using the briefcase OCT system, an image of layered tape (S-9783, 3M 810 Scotch Tape) was obtained by translating the sample using a motorized translation stage. During imaging, the probe was fixed on a mount and a sample was placed on a sample holder. Figure 6(a) shows a cross-sectional image of the layered tape wherein $>15$ layers are clearly visualized. The dimensions of the acquired B-mode image were $7 \mathrm{~mm} \times 1.5 \mathrm{~mm}$, with 512 axial points in depth along each A-scan, and with 1000 A-scans of data making up the cross-sectional B-mode image. The image of the layered tape shows that the current imaging resolution and depth are sufficient for imaging biological samples.

We further imaged human skin sites including the palm region, nail fold, and fingertip. The dimensions of the acquired B-mode images were the same as for the layered tape. Human subject imaging was performed under a protocol approved by the Institutional Review Board at the University of Illinois at Urbana-Champaign. Figures 6(b)-6(d) show representative cross-sectional images of these sites. Our system was able to resolve intricate features in the palm and fingertip such as the stratum corneum, stratum spinosum, sweat ducts, and the dermis layer. The image of the nail fold revealed features such as the epidermis, dermis, and nail plate. The missing A-lines in Fig. 6(d) were due to the movement of the fingertip during acquisition ( 10-mm scan). Acquisition of the B-mode images using a motorized stage was done to compare image quality with cross-sectional images assembled using the manual scanning approach.

\subsection{Manual Scanning: Mosaicking and Cross-Sectional Imaging Approach}

After implementing manual scanning and real-time mosaicking, we further tested the feasibility of this approach by once again imaging in vivo human skin. Figure 7 shows the OCT and surface image mosaic of skin from the human palm. The real-time surface mosaicking showed precise image registration, resolving the papillary ridges (fingerprint) of the skin in the palm region [Fig. 7(a)]. We also observed the misregistration of the mosaic in Fig. 7(a) at the angled surface of the palm. This could be due to the defocused image limiting the accuracy of the registration algorithm. In the present study, we had implemented a sharpness-based metric to eliminate out-of-focus images and saved real-time mosaics as they were formed.

Figure 7(b) shows the plot of the OCT beam position during the real-time scan. This position plot was based on the measured offset value of the image registration corresponding to the acquired A-scan position. The cross-sectional beam path image generated for the integrated path of the OCT beam position is shown in Fig. 7(c). The represented image shows well-differentiated structures such as the stratum corneum, sweat ducts, and the dermis. This image has a lateral dimension 


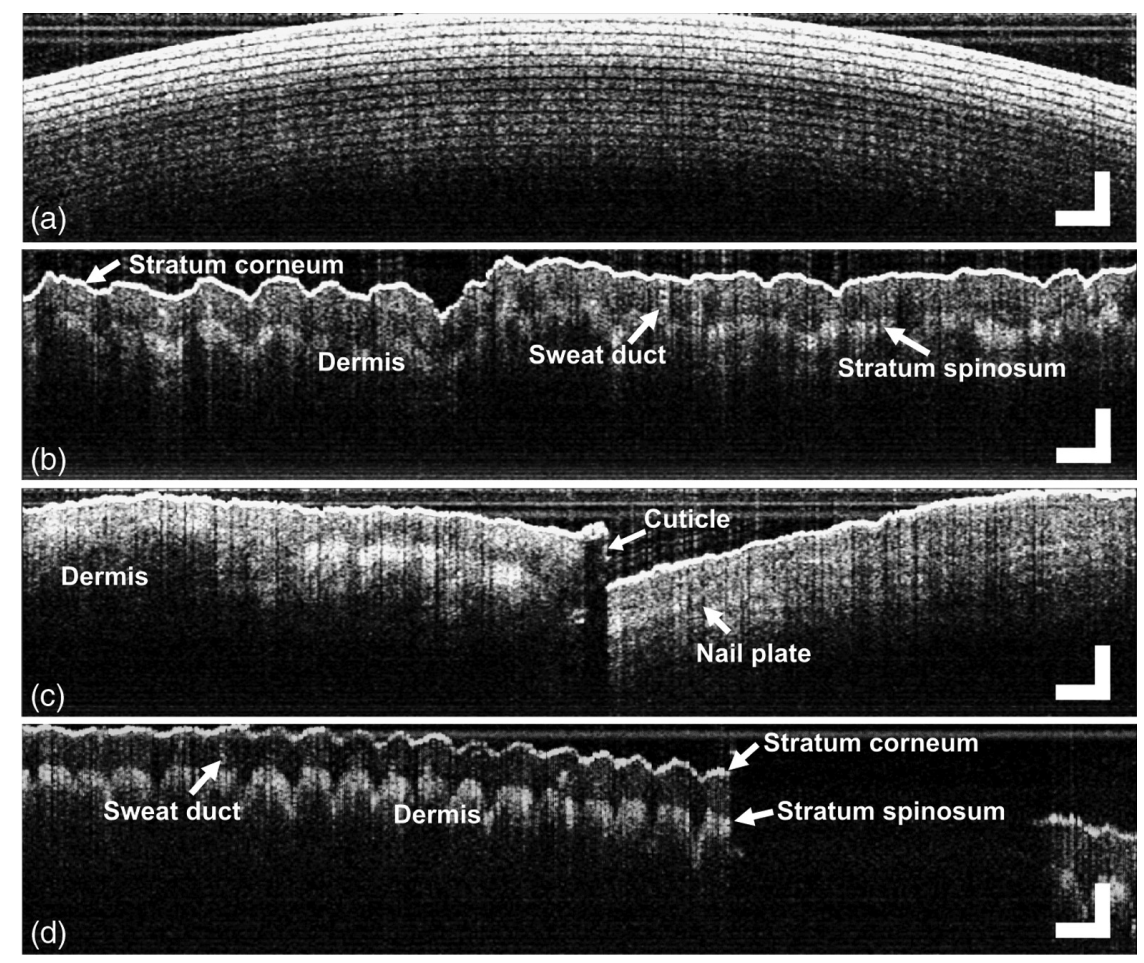

Fig. 6 Representative cross-sectional images obtained by translating the sample with a motorized stage. (a) Reconstructed OCT image of a layered roll of tape, (b) in vivo imaging of the human palm region, (c) fingernail plate and fold, and (d) fingertip. The scale bar represents $500 \mu \mathrm{m}$.

(a)

$$
\text { Surface mosaic }
$$

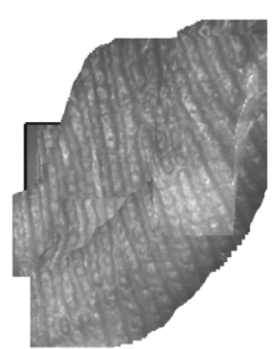

OCT beam position

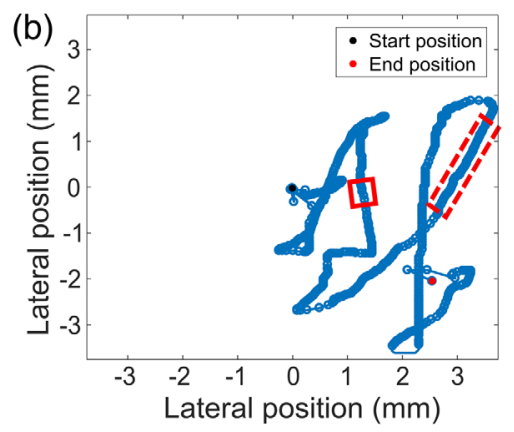

(d)
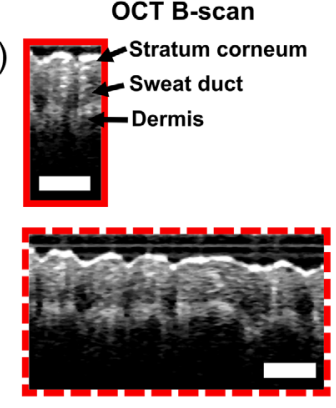

Cross-sectional beam path image

(c)

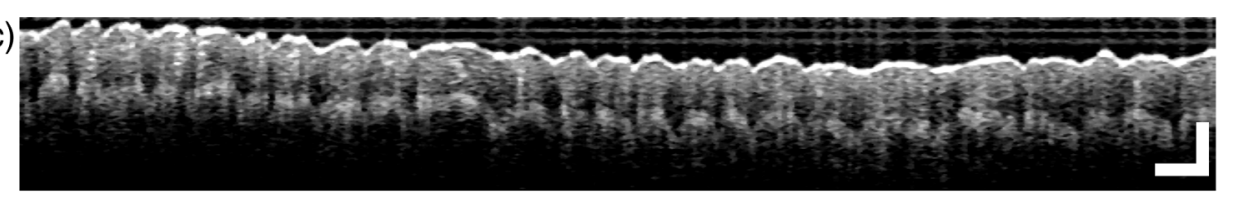

Fig. 7 Cross-sectional beam path image and surface mosaic of the in vivo human palm. (a) Surface mosaic of the palm region with the corresponding OCT beam position shown in (b). (c) Cross-sectional image of the palm constructed from the corresponding OCT beam path. (d) Representative B-scans are formed by extracting adjacent A-scans from a straight path in the manual scan shown by the solid and dashed box outlines. The scale bar represents $500 \mu \mathrm{m}$.

of over $10 \mathrm{~mm}$ and was obtained from both dimensions ( $X$ and $Y)$ in the beam path position. This system allows a user to manually and arbitrarily scan larger FOVs. Furthermore, the recorded path of the beam position enables the construction of a thickness map that could be overlaid on the surface image mosaic. Figure 7(d) shows representative B-scans obtained from the same dataset at two different straight paths highlighted in Fig. 7(b). The B-scans obtained from this manual scanning approach were comparable with the B-scans obtained using the motorized stage. The results from imaging the palm show that our system can clearly resolve microstructural features in scattering samples.

We further imaged the human fingertip and forearm regions. The OCT and surface image mosaics from both tissue sites 


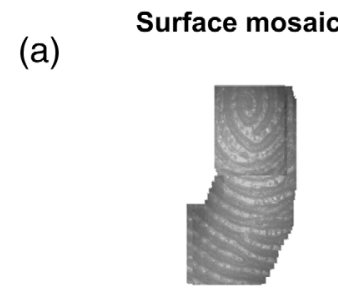

(b)

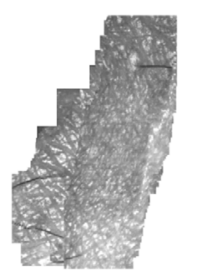

(c)

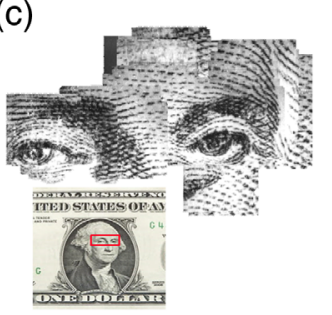

OCT beam position

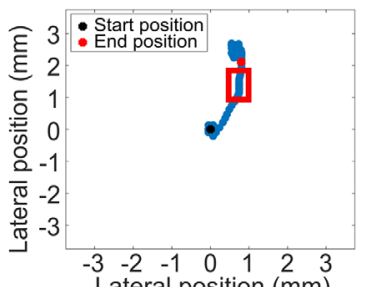

Lateral position $(\mathrm{mm})$

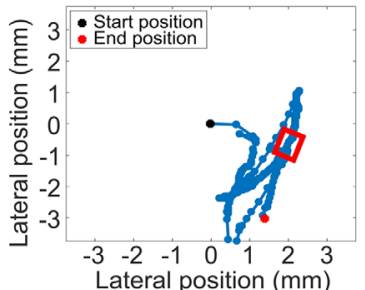

Lateral position ( $\mathrm{mm})$

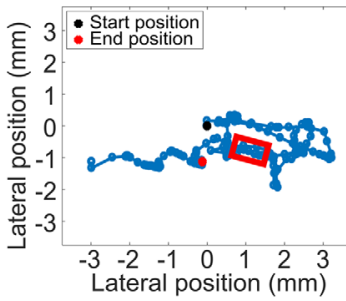

Cross-sectional beam path image
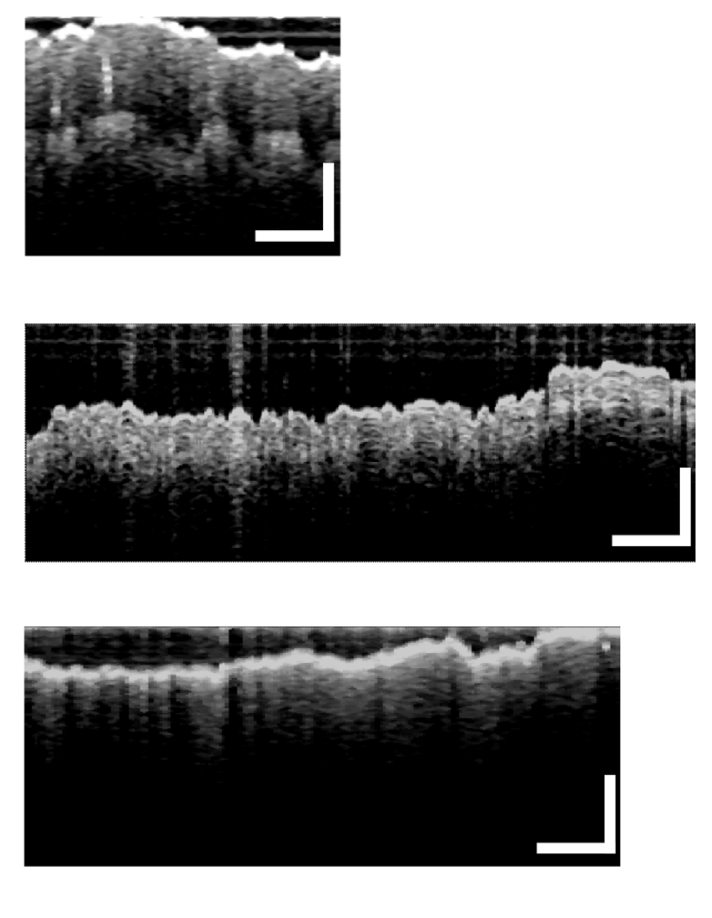

OCT B-scan
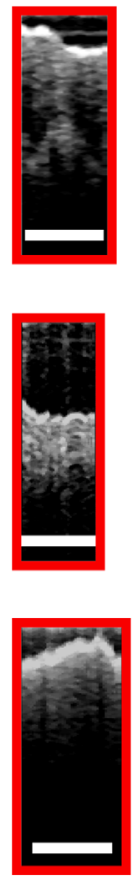

Fig. 8 Cross-sectional beam path images and surface mosaics of human skin tissue sites and a dollar bill. Surface mosaics, OCT beam position plots, and corresponding cross-sectional beam path and B-scan images are shown for (a) human fingertip, (b) forearm skin, and (c) US dollar bill. The representative OCT B-scan images are extracted from the straight path regions as shown by the solid box outlines. The scale bars represent $500 \mu \mathrm{m}$.

resolved optimal surface features and depth-resolved structures as shown in Figs. 8(a) and 8(b). Cross-sectional images and the corresponding surface mosaic of a US dollar bill are shown in Fig. 8(c). A dollar bill was imaged to highlight the accuracy of the image registration and the real-time mosaicking algorithm as fine features can be resolved in the surface image mosaic. The cross-sectional image of the dollar bill, however, lacked structural features, and largely only a surface reflection could be visualized by OCT. From these results, it can be concluded that the briefcase OCT system with manual scanning and real-time mosaicking can accurately and simultaneously construct surface and depth-resolved cross-sectional images in real time.

\section{Discussion}

A portable, low-cost, briefcase OCT system has been developed for primary care and point-of-care imaging applications. This system includes a handheld probe with a surface imaging module to enable both real-time surface image mosaicking along with fast manual lateral scanning for imaging large FOVs. This functionality was achieved in a system weighing $9 \mathrm{~kg}$, constructed with off-the-shelf components at a total cost of $\sim$ USD8000. This low cost was achievable with the incorporation of inexpensive yet efficient components and scanning methods. This includes modifications to a low-cost OEM spectrometer, and the use of an uncooled SLD. Our system also included a manual scanning approach that obviates the need for a lateral scanning system such as a galvanometer or MEMS scanner, and their associated drivers and power supplies. The use of this manual scanning approach along with a USBbased detector eliminated the need for frame grabber and data acquisition boards. This combination of features enabled visualization of both subsurface structure and surface features at large FOVs in real time.

The developed briefcase OCT system has significant advantages over low-cost TD-OCT technology in terms of speed and sensitivity. Our developed system demonstrated a higher line rate of $10 \mathrm{kHz}$, which is higher than the reported low-cost TD-OCT technology. ${ }^{20,37}$ In these terms, the use of FD-OCT technology opens a broad range of options for low-cost OCT systems. Researchers globally are striving to miniaturize SDOCT and SS-OCT on a silicon chip. Nonetheless, miniature OCT systems remain in early developmental stages, and are currently not integrated in a complete OCT system. In terms of portability, this briefcase OCT system is comparable with a recently reported portable and battery-powered OCT system, which was demonstrated for characterizing crop health in the field. ${ }^{38}$ The battery-powered backpack OCT system was based on spectral-domain detection and included the OCT system in an 8-kg backpack with dimensions of $70 \mathrm{~cm} \times 50 \mathrm{~cm} \times 15 \mathrm{~cm}$. Although the backpack OCT system was exceptionally portable with its battery power, it was limited by the use of high-priced components, in contrast to our system that was focused on lowcost, economical, yet efficient components.

In this study, we demonstrated the imaging capability of the briefcase OCT system by imaging the in vivo human fingertip, palm, and forearm regions. The B-scans obtained from the manually scanned tissue sites were comparable with those from the same skin tissue sites but obtained using a motorized translation stage. The results show that our system enables visualization of internal microstructures of highly scattering tissues at high 
resolution and at high speed. The surface image mosaics generated using our system can accurately register the sample features (as shown by the dollar bill mosaic [Fig. 8(c)]. The OCT images shown in (Figs. 6-8) were constructed without any frame averaging and confirm that the system sensitivity and resolution are suitable for imaging.

Despite the noted advantages, the present study has several limitations. The developed briefcase OCT system was able to acquire spectra at $10 \mathrm{kHz}$, which is at a lower rate compared to newer SD-OCT or SS-OCT systems. However, trade-offs were made in designing this low-cost system, such as limiting the high-end performance that is provided by modern, high-end, and more costly OCT systems. Ongoing work is aiming to improve the speed and sensitivity of the existing briefcase OCT system. An earlier report compared the performance of OCT between 8- and 14-bit operations and found subtle changes in the magnitude images. ${ }^{39}$ The CCD detector used in this system can also acquire spectra at $25 \mathrm{kHz}$ with an eight-bit mode operation, which will likely offer the potential to improve imaging speed with perhaps only a minimal change in image quality. Though the system had an imaging range of $2.7 \mathrm{~mm}$, detection of the depth signal was restricted to $1.5 \mathrm{~mm}$, due to the sensitivity roll-off. Further optimization of the spectrometer by machining a new housing to better position the camera will further enhance the sensitivity and imaging range, as well as by implementing full-range OCT approaches. ${ }^{40}$ The full-range method will double the imaging range (to $\sim 5.4 \mathrm{~mm}$ ), enabling positioning of the sample at zero OPD, where the system has highest sensitivity.

The cross-sectional images were reconstructed based on a manual scanning approach by measuring the degree of correlation between sequential A-scans. This technique has certain limitations on the speed of image acquisition as the sampling factor must be higher for accurate image reconstruction. ${ }^{32}$ Hence, the velocity of the probe needs to be constrained so that the sample is sufficiently oversampled. Moving faster than the threshold will result in undersampling. A possible extension of this algorithm could be to detect undersampling by comparing the cross-correlation coefficients of adjacent A-scans against a lower threshold value. Interpolation algorithms can also be used to estimate missing A-scans. Additionally, a potential extension could be to use the phase information to detect lateral displacement. Reports have shown that incorporation of a speckle model into the decorrelation function enables correlation of the lateral displacement between adjacent A-scans, providing real-time, distortion-free images. ${ }^{41,42}$ The reported approach was computationally intensive and less suitable for the briefcase OCT system, especially due to the standard-performance laptop. Our group also reported a sensor-based approach to track the position of the imaging probe with respect to the sample, which provides motion-artifact-free cross-sectional images. ${ }^{43}$ Further optimization of the manual scanning method is possible to make it more compatible for low-cost briefcase OCT systems. During imaging, the probe was scanned laterally without much movement of the probe in depth (or height above the surface), and the correlations between sequential A-scans were measured. Probe height movement could also be reduced using a spacer attached to the tip of the probe. This fixed spacer would be helpful for all users, although this approach makes the probe contact-based. The developed briefcase OCT system was originally designed based on our experience with ear/TM imaging. Our group has previously used high-end 800-nm OCT systems for TM imaging, which has led to several new findings. ${ }^{17-19,44}$ Here, we have shown representative skin images to test the applicability and feasibility of our system. The presented system, however, was not fully optimized for TM imaging, and efforts are being made to do so. Furthermore, this lowcost spectral-domain OCT system was attainable due to the availability of low-cost silicon detectors (at $800 \mathrm{~nm}$ ). Currently, there are no suitable low-cost sources available for wavelengths around $1300 \mathrm{~nm}$, as InGaAs detectors are comparably more expensive.

In the future, it is expected that the cost of this briefcase OCT can be further reduced by at least a factor of 2 using more OEM components and an integrated computer and low-cost display (versus an off-the-shelf laptop). The weight of the developed briefcase OCT system is also expected to be reduced by a factor of 2 by replacing the metal base plate, enclosure plate, and vertical plates with a custom-designed 3-D printed case for mounting and housing all optics and electronics.

\section{Conclusion}

There has been significant interest in developing low-cost and miniature OCT systems for primary care applications, as well as for specialists in areas such as otolaryngology, ophthalmology, dermatology, and dentistry. Here, we reported the development and evaluation of a portable, low-cost, briefcase OCT system that is suitable for use in primary care and point-of-care applications, in many specialty areas of medicine, for biological field work, and for nondestructive testing of materials. With lower cost components becoming more and more available, and with the potential for an even smaller form factor, this OCT system technology has the potential to further broaden the applications of OCT.

\section{Disclosures}

S.A. Boppart is cofounder and chief medical officer of PhotoniCare, Inc., which is developing optical imaging systems for the ear, and Diagnostic Photonics, Inc., which is developing optical imaging systems for cancer detection. S.A.B. also reports intellectual property owned by the Massachusetts Institute of Technology and the University of Illinois at Urbana-Champaign related to OCT, and licensed to commercial entities, that is related to the technology and analysis methods described in some parts of this paper. Other authors report no disclosures or conflicts of interest.

\section{Acknowledgments}

Research in this publication was supported by the National Institute for Biomedical Imaging and Bioengineering and the National Cancer Institute of the National Institutes of Health under Award Nos. R01EB013723 and R01CA213149, respectively. One-hundred percent $(\$ 125,000)$ of the total project costs were financed with federal money and zero percent $(\$ 0.00)$ of the total costs were financed by nongovernmental sources. The content is solely the responsibility of the authors and does not necessarily represent the official views of the National Institutes of Health. The authors thank Greggory Len Bennett and Scott A. McDonald from the Electrical and Computer Engineering Department machine shop, University of Illinois at Urbana-Champaign, for their assistance in constructing mechanical mounts. The authors also thank Jose RicoJimenez and Andrew Bower from the Beckman Institute for Advanced Science and Technology, University of Illinois at 
Urbana-Champaign, for their suggestions on designing the LabVIEW acquisition program. Finally, the authors thank Heidi Olsen from Ibsen Photonics for providing information regarding the I-MON 835 OEM unit.

\section{References}

1. D. Huang et al., "Optical coherence tomography," Science 254(5035), 1178-1181 (1991).

2. R. Leitgeb, C. Hitzenberger, and A. Fercher, "Performance of Fourier domain vs. time domain optical coherence tomography," Opt. Express 11(8), 889-894 (2003).

3. M. A. Choma et al., "Sensitivity advantage of swept source and Fourier domain optical coherence tomography," Opt. Express 11(18), 21832189 (2003).

4. E. A. Swanson and J. G. Fujimoto, "The ecosystem that powered the translation of OCT from fundamental research to clinical and commercial impact," Biomed. Opt. Express 8(3), 1638-1664 (2017).

5. J. Fujimoto and E. Swanson, "The development, commercialization, and impact of optical coherence tomography," Invest. Ophthalmol. Vis. Sci. 57(9), OCT1-OCT13 (2016).

6. W. Jung et al., "Handheld optical coherence tomography scanner for primary care diagnostics," IEEE Trans. Biomed. Eng. 58(3), 741-744 (2011).

7. R. L. Shelton et al., "Optical coherence tomography for advanced screening in the primary care office," J. Biophotonics 7(7), 525-533 (2014).

8. G. L. Monroy et al., "Clinical translation of handheld optical coherence tomography: practical considerations and recent advancements," J. Biomed. Opt. 22(12), 121715 (2017).

9. S. J. Erickson-Bhatt et al., "Real-time imaging of the resection bed using a handheld probe to reduce incidence of microscopic positive margins in cancer surgery," Cancer Res. 75(18), 3706-3712 (2015).

10. R. M. Nolan et al., "Intraoperative optical coherence tomography for assessing human lymph nodes for metastatic cancer," BMC Cancer 16(1), 144 (2016).

11. K. J. Mesa et al., "Intraoperative optical coherence tomography for soft tissue sarcoma differentiation and margin identification," Lasers Surg. Med. 49(3), 240-248 (2017).

12. F. LaRocca et al., "In vivo cellular-resolution retinal imaging in infants and children using an ultracompact handheld probe," Nat. Photonics 10(9), 580-584 (2016).

13. C. T. Nguyen et al., "Noninvasive in vivo optical detection of biofilm in the human middle ear," Proc. Natl. Acad. Sci. 109(24), 9529-9534 (2012).

14. G. L. Monroy et al., "Noninvasive depth-resolved optical measurements of the tympanic membrane and middle ear for differentiating otitis media," The Laryngoscope 125(8), E276-E282 (2015).

15. G. L. Monroy et al., "Noninvasive in vivo optical coherence tomography tracking of chronic otitis media in pediatric subjects after surgical intervention," J. Biomed. Opt. 22(12), 121614 (2017).

16. G. L. Monroy et al., "Non-invasive optical assessment of viscosity of middle ear effusions in otitis media," J. Biophotonics 10(3), 394-403 (2017).

17. R. L. Shelton et al., "Quantitative pneumatic otoscopy using a lightbased ranging technique," J. Assoc. Res. Otolaryngol. 18(4), 555568 (2017).

18. J. Won et al., "Pneumatic low-coherence interferometry otoscope to quantify tympanic membrane mobility and middle ear pressure," Biomed. Opt. Express 9(2), 397-409 (2018).

19. P. Pande et al., "A mosaicking approach for in vivo thickness mapping of the human tympanic membrane using low coherence interferometry," J. Assoc. Res. Otolaryngol. 17(5), 403-416 (2016).

20. P. Pande et al., "Low-cost hand-held probe for depth-resolved lowcoherence interferometry," Biomed. Opt. Express 8(1), 338-348 (2017).

21. R. Dsouza et al., "Dermascope guided multiple reference optical coherence tomography," Biomed. Opt. Express 5(9), 2870-2882 (2014).

22. M. J. Leahy et al., "The how and why of a $\$ 10$ optical coherence tomography system," Proc. SPIE 9697, 96970T (2016).

23. P. M. McNamara et al., "Development of a first-generation miniature multiple reference optical coherence tomography imaging device," J. Biomed. Opt. 21(12), 126020 (2016).
24. W. Drexler, "Horizon 2020 project aims to transform optical coherence tomography," http://optics.org/news/7/6/19 (6 March 2018).

25. B. I. Akca et al., "Miniature spectrometer and beam splitter for an optical coherence tomography on a silicon chip," Opt. Express 21(14), 16648-16656 (2013).

26. G. Yurtsever et al., "Ultra-compact silicon photonic integrated interferometer for swept-source optical coherence tomography," Opt. Lett. 39(17), 5228-5231 (2014).

27. S. Schneider et al., "Optical coherence tomography system massproducible on a silicon photonic chip," Opt. Express 24(2), 15731586 (2016).

28. V. D. Nguyen et al., "Spectral domain optical coherence tomography imaging with an integrated optics spectrometer," Opt. Lett. 36(7), 1293-1295 (2011).

29. V. D. Nguyen et al., "Integrated-optics-based swept-source optical coherence tomography," Opt. Lett. 37(23), 4820-4822 (2012).

30. Z. Wang et al., "Silicon photonic integrated circuit swept-source optical coherence tomography receiver with dual polarization, dual balanced, in-phase and quadrature detection," Biomed. Opt. Express 6(7), 2562-2574 (2015).

31. S. Kim et al., "Design and implementation of a low-cost, portable OCT system," Biomed. Opt. Express 9(3), 1232-1243 (2018).

32. A. Ahmad et al., "Cross-correlation-based image acquisition technique for manually-scanned optical coherence tomography," Opt. Express 17(10), 8125-8136 (2009).

33. "American National Standard for Safe Use of Lasers ANSI Z136.1," Laser Institute of America, Orlando, Florida (2007).

34. M. Guizar-Sicairos, S. T. Thurman, and J. R. Fienup, "Efficient subpixel image registration algorithms," Opt. Lett. 33(2), 156-158 (2008).

35. N. Bedard et al., "Real-time video mosaicing with a high-resolution microendoscope," Biomed. Opt. Express 3(10), 2428-2435 (2012).

36. T. Vercauteren et al., "Real time autonomous video image registration for endomicroscopy: fighting the compromises," Proc. SPIE 6861, $68610 \mathrm{C}(2008)$.

37. K. Neuhaus et al., "Simultaneous en-face imaging of multiple layers with multiple reference optical coherence tomography," J. Biomed. Opt. 22(8), 086006 (2017).

38. R. E. Wijesinghe et al., "Optical coherence tomography-integrated, wearable (backpack-type), compact diagnostic imaging modality for in situ leaf quality assessment," Appl. Opt. 56(9), D108-D114 (2017).

39. Z. Lu, D. K. Kasaragod, and S. J. Matcher, "Performance comparison between 8- and 14-bit-depth imaging in polarization-sensitive sweptsource optical coherence tomography," Biomed. Opt. Express 2(4), 794-804 (2011).

40. M. Wojtkowski et al., "Full range complex spectral optical coherence tomography technique in eye imaging," Opt. Lett. 27(16), 1415-1417 (2002).

41. X. Liu, Y. Huang, and J. U. Kang, "Distortion-free freehand-scanning OCT implemented with real-time scanning speed variance correction," Opt. Express 20(15), 16567-16583 (2012).

42. X. Liu et al., "Robust spectral-domain optical coherence tomography speckle model and its cross-correlation coefficient analysis," J. Opt. Soc. Am. A 30(1), 51-59 (2013).

43. P. Pande et al., "Sensor-based technique for manually scanned handheld optical coherence tomography imaging," J. Sens. 2016, 1-7 (2016).

44. G. L. Monroy et al., "Direct analysis of pathogenic structures affixed to the tympanic membrane during chronicotitis media," Otolaryngol. Head Neck Surg. 159(1), 117-126 (2018).

Roshan Dsouza received his MS degree in photonics from Manipal University, India, in 2011 and his PhD in biomedical optics from the National University of Ireland, Galway, in 2016. Currently, he is a postdoctoral researcher at the Beckman Institute for Advance Science and Technology, University of Illinois at Urbana-Champaign. His research interests include the development of low-cost imaging devices and biophotonics applications in low-resource settings.

Jungeun Won received her BS degree in biomedical engineering from the University of Rochester in 2015 and received her MS degree in bioengineering from the University of Illinois at Urbana-Champaign in 2017. She is a graduate student working toward her PhD in bioengineering in the Biophotonics Imaging Laboratory, working under the guidance of Prof. Stephen Boppart. Her research interests include 
developing optical imaging systems and analytic methods to improve medical diagnosis.

Guillermo L. Monroy received his BS degree in computer engineering at the University of Illinois at Urbana-Champaign in 2011 and continued for his MS degree in bioengineering in 2013 and his PhD in 2018. His thesis work and current interests focus on the clinical translation of advanced biomedical instrumentation and imaging systems to improve diagnosis and treatment in health care.

Darold R. Spillman Jr. received his associate of arts degree in human resources from the Community College of the Air Force, Maxwell Air Force Base, Georgia, in 2005 and his BA degree in general studies from Eastern Illinois University, Charleston, Illinois, in 2015. He served in the United States Air Force from 1984-2007. He was a 1993 Presidential Point of Light Award nominee and was spotlighted in the Inside Illinois publication.

Stephen A. Boppart received his BS and MS degrees in electrical engineering from the University of Illinois at Urbana-Champaign (UIUC), his PhD in electrical and medical engineering from MIT, and his MD from Harvard Medical School. He is the Abel Bliss professor of engineering, head of the Biophotonics Imaging Laboratory, and director of the Center for Optical Molecular Imaging at UIUC. He is a fellow of AAAS, SPIE, IEEE, OSA, BMES, and AIMBE. 